\title{
Helico-conical optical beams self-heal
}

\author{
N. Hermosa, ${ }^{1, *}$ C. Rosales-Guzmán, ${ }^{1}$ and J. P. Torres ${ }^{1,2}$ \\ ${ }^{1}$ ICFO_Institut de Ciencies Fotoniques, Technical University of Catalonia, Mediterranean Technology Park, \\ Castelldefels (Barcelona) 08860, Spain \\ ${ }^{2}$ Department of Signal Theory and Communications, Technical University of Catalonia, Barcelona 08034, Spain \\ *Corresponding author: nathaniel.hermosa@icfo.es
}

Received November 26, 2012; revised January 7, 2013; accepted January 7, 2013; posted January 9, 2013 (Doc. ID 180626); published February 1, 2013

An optical beam is said to be self-healing when, distorted by an obstacle, the beam corrects itself upon propagation. In this Letter we show, through experiments supported by numerical simulations, that Helico-conical optical beams self-heal. We observe the strong resilience of these beams with different types of obstructions, and relate this to the characteristics of their transverse energy flow. (c) 2013 Optical Society of America

OCIS codes: $140.3300,260.6042,260.0260,070.2580$.

The study of the self-healing properties of beams is of great interest in optics [1-6]. An optical beam is said to be self-healing when, after propagation, its transverse intensity profile is hardly affected by a small perturbation-a block - that has been placed in its path [1-5]. The surge of interest in self-healing beams is bouyed mainly by its range of applications; self-healing can be advantageous, for instance, in beam propagation through scattering and turbulent media, and in optical manipulation $[\underline{7}, \underline{8}]$.

Optical beams that exhibit self-healing include Bessel beams (BBs) [6,7,9], caustic beams [4], Airy beams [1,8], Pearcey beams [2], the nonparaxial Mathieu and Weber accelerating beams [10], and some forms of LaguerreGaussian (LG) beams [므. In the case of the BBs and Airy beams, self-healing happens at a relatively small propagation distance, while LG beams self-heal at a distance of the order of the Rayleigh length $[2,3]$. Self-healing is independent of the diffracting nature of the beams, as shown by caustic [4] and LG beams [3].

In this Letter, we present another set of beams that self-heal: the Helico-conical optical beams (HCOBs). The main difference between these beams and other self-healing beams is the nonseparability of their radial and azimuthal phases [11]. HCOBs posses a phase $\psi$ that is the product of a helical phase and a conical phase: $\psi(r, \theta)=\ell \theta\left(K-r / r_{0}\right)$, where $\ell$ is the winding number around the azimuth angle $\theta, r_{0}$ normalizes the radial coordinate $r$, and $K$ takes either the value 0 or 1 . At the far field, the intensity profile of these beams resembles a spiral, with $K=1$ HCOBs having a more pronounced head near the center of the beam axis compared with the $K=0$ HCOBs. Recently, a $K=0$ HCOB was reported to cause a spiral motion to a particle along its path $[12,13]$, a three dimensional motion that combines phase gradient with intensity gradient forces [14].

It could be argued that, since HCOBs have conical phases, they should behave similar to BBs. In fact, the HCOBs are more likely to be compared with fractional higher-order BBs because of their similar intensity distributions [15]. Joint to this is the fact that HCOBs consist of strings of optical vortices upon propagation [16].

However, the far-field intensity pattern of experimentally generated BBs, or any superposition of it, resembles a circle or a $\delta$-ring [17], while HCOBs are spirals in the far field, and not rings when compared to BBs [11]. An important question then arises: Can HCOBs self-heal?

Here, we provide evidence that an $\mathrm{HCOB}$ reconstructs its intensity profile at a relatively short propagation distance after a small perturbation is placed in its path. We observe how the beam reconstructs for different values of $\ell$ and for different block sizes. Since the phase of the HCOB is not rotationally symmetric, we also note how the beam reconstruct when we change the orientation of the obstructing block. We then compare our experimental results with numerical simulations. Finally, we look at the transverse energy flow of the beam and relate it to its self-healing property.

We generate the HCOBs using a spatial light modulator (SLM) [18,19]. Figure 1(a) shows the experimental setup. A collimated $\mathrm{He}-\mathrm{Ne}$ laser $(\lambda=632.8 \mathrm{~nm})$ beam impinges onto an SLM (Hamamatsu LCOS-SLM) encoded with a computer-generated hologram. The holograms $\left(r_{0}=2.5 \mathrm{~mm}\right)$ are calculated from the phase of the HCOBs. A carrier frequency is added to separate the beam of interest. We observe the beam after propagation from the SLM while varying $\ell$, and the size and orientation of the block. We imitate the presence of a block by means of an incomplete hologram [see Fig. 1(b)]. This is done for better control of the size of the block. The block size corresponds to an angular fraction $\Delta \theta$. We then capture the intensity pattern with a charged couple device (CCD) camera attached to a computer after $14 \mathrm{~cm}$ of propagation. Using the angular spectrum method [20], we compare our results with numerical simulations. Figure 2 shows the intensity profiles of the unblocked and blocked HCOBs with $\ell=50$ and $\Delta \theta=\pi / 3$. The intensity
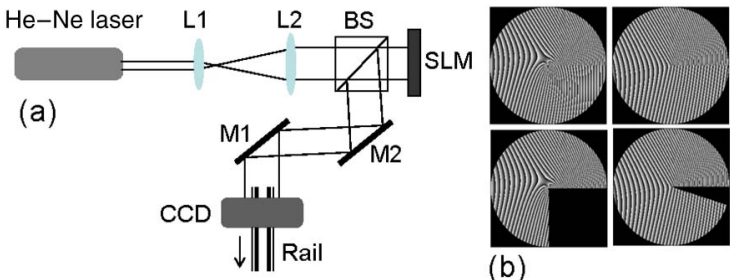

(b)

Fig. 1. (Color online) (a) Experimental setup. Holograms are encoded onto a SLM. (b) Samples of unblocked (above) and blocked (below) holograms are shown. L1 and L2 are collimating lenses while M1 and M2 are mirrors for alignment. BS is beam splitter. 

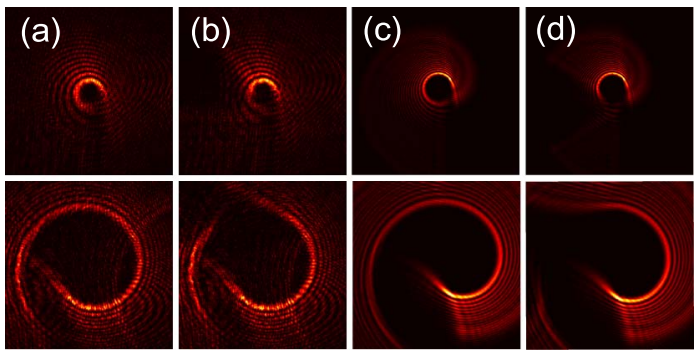

Fig. 2. (Color online) Intensity profiles comparing reconstruction of blocked (b), (d) $\ell=50$ HCOBs after $14 \mathrm{~cm}$ propagation with beams that are not blocked (a), (c). (a) and (b) are obtained from experiments while (c) and (d) are from numerical simulations. The top images are for $K=0$ while the bottom ones are for $K=1$. The block size is $\Delta \theta=\pi / 3$.

patterns, not measured in the far field, scale with the value of $\ell$, similar to what is shown in [11].

The similarities between blocked and unblocked beams are quantified for both experiments and simulations using 2D image correlation [21]. The value of the correlation coefficient ranges from 0 for nonidentical beams to 1 for identical beams. We emphasize, however, that the correlation coefficient only gives a trend. It is not an exact measure of the quality of the beam reconstruction, especially in our case, wherein it is difficult to separate the generated beam from the adjacent diffraction orders.

The correlation coefficient changes with the value of $\ell$ as shown in Fig. 3(a). The block size is $\pi / 3$ and the orientation of the block is shown as an inset. As the $\ell$ value increases, the correlation coefficient also increases in both the experiment and numerical simulations, with the $K=0$ HCOBs having higher correlation coefficients than the $K=1$ HCOBs with the same $\ell$, at the same propagation distance.

The block size affects the reconstruction of the HCOBs as shown in Fig. 3(b). The $K=0$ HCOBs reconstruct faster than the $K=1$ HCOBs, given the same block size and the same propagation distance. We notice that the beam with a $\pi$ block size (not shown) gives a very low correlation coefficient, which is consistent with the results previously reported for other self-healing beams $[\underline{4}, 6]$. The HCOB reconstruction also depends on the orientation of the block, as shown in Fig. 3(c). The block size is $\Delta \theta=\pi / 4$ and the orientation of the block is shown below the plot. This is expected since the intensity and the phase of the beam are not rotationally symmetric.

In another experiment, we block the HCOBs with a $0.38 \mathrm{~mm}$ opaque strip, and observe how the HCOBs reconstruct upon propagation. We restrict our measurement to distances below $r_{0}^{2} / \ell \lambda$, since we notice that beyond this distance, the HCOBs' intensity profiles change more rapidly [22]. Figure 4 shows the experimental and numerical results, with an opaque strip placed $16 \mathrm{~cm}$ after the SLM. Placing the CCD at different distances, we observe that the HCOBs self-heal as the beams propagate. The shadow of the block moves in a rotatory manner, similar to the self-healing of higher order BBs. However, unlike BBs, HCOBS slightly rotate and expand.
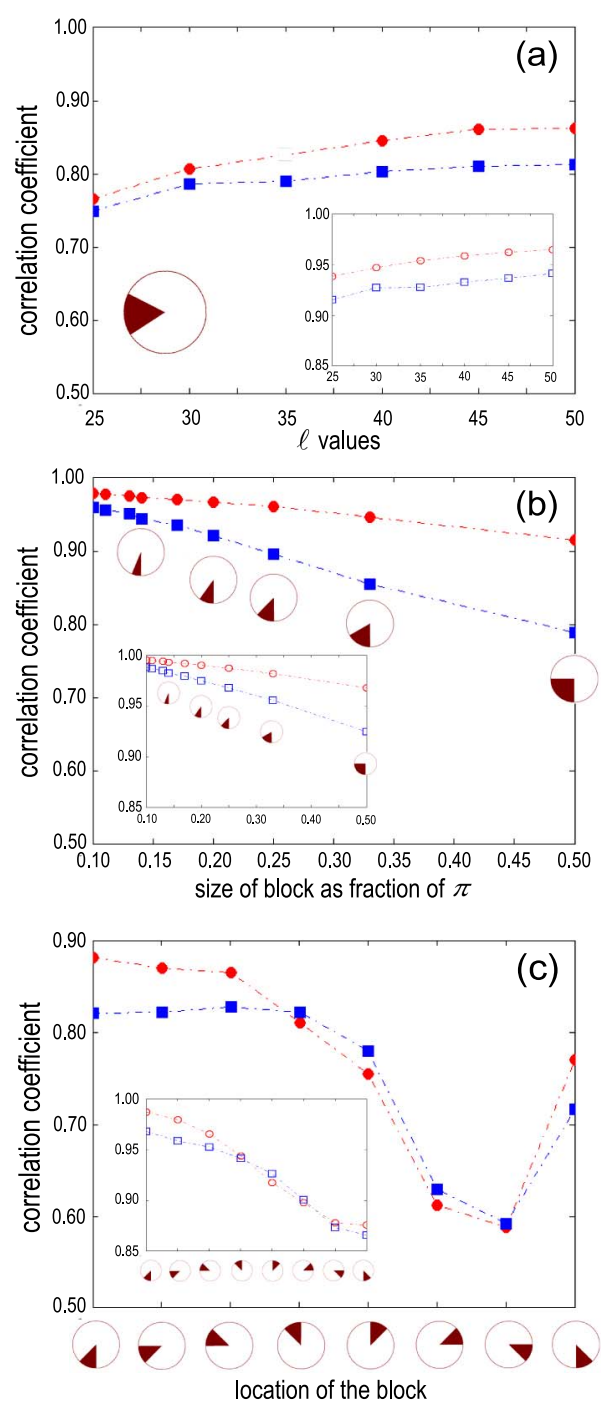

Fig. 3. (Color online) (a) Correlation for different values of $\ell$. (b) Correlation with different block sizes. (c) Correlation for different block orientations. Numerical simulation results are placed as inset. $K=0$ uses circles, while $K=1$ uses squares.

Figure 5 shows the transverse energy flow of an $\ell=30$ $K=0 \mathrm{HCOB}$, calculated numerically [23]. Similar images can be obtained for $K=1 \mathrm{HCOBs}$. The direction of the energy flow traces a curved path (shown as white arrows in the figure). Even with the block, the direction of the
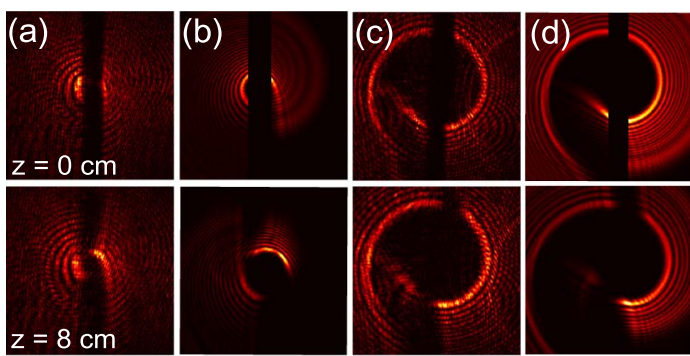

Fig. 4. (Color online) A $0.38 \mathrm{~mm}$ strip is placed at the path of a $\ell=40 \mathrm{HCOB}, 16 \mathrm{~cm}$ after the SLM. (a) and (c) are experimental results while (b) and (d) are numerical simulations. Top images are obtained right after the block while the bottom images are after $8 \mathrm{~cm}$ of propagation. (a), (b) and (c), (d) are $K=0$ and $K=1$ HCOBs, respectively. 

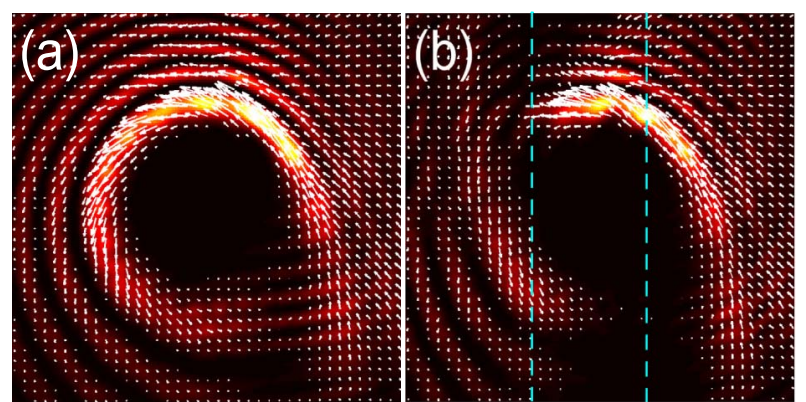

Fig. 5. (Color online) Transverse energy flow for $K=0$ HCOBs with $\ell=30$ for (a) no block and (b) blocked located $16 \mathrm{~cm}$ after the SLM. Both beams propagate a total distance of $20 \mathrm{~cm}$. Arrow direction is the direction of the energy flow while its length is the magnitude. Dashed lines denote the original position of the block.

energy flow is unaltered, and so the energy flows from the surrounding areas to the blocked area [24]. Since the energy and the energy flow are greater at the upper section, the beam reconstructs faster in this part. In addition, the transverse energy flow is greater for larger $\ell$ values which translates to faster reconstruction (not shown). This suggests that the transverse energy flow is the reason that the beam reconstructs.

In summary, we have shown experimentally that HCOBs self-heal and we have supported our results with numerical simulations. The intensity profile reconstructs under different circumstances: by varying the size and the orientation of the obstructing block, as well as by changing the $\ell$ value of the HCOBs. We observe how the beams heal as they propagate, and we link our results with the transverse energy flow within the beam. The resilience of these beams could ease its applicability in optical trapping and manipulation.

This work was supported by the Government of Spain (FIS2010-14831), PHORBITECH (grant number: 255914), and Fundacio Privada Cellex Barcelona. The authors would like to thank V. Rodríguez-Fajardo and L. J. Salazar-Serrano for useful discussions.

\section{References and Notes}

1. J. Broky, G. Siviloglou, A. Dogariu, and D. Christodoulides, Opt. Express 16, 12880 (2008).

2. J. Ring, J. Lindberg, A. Mourka, M. Mazilu, K. Dholakia, and M. Dennis, Opt. Express 20, 18955 (2012).

3. P. Vaity and R. P. Singh, Opt. Lett. 36, 2994 (2011).

4. M. Anguiano-Morales, A. Martínez, M. Iturbe-Castillo, S. Chávez-Cerda, and N. Alcalá-Ochoa, Appl. Opt. 46, 8284 (2007).

5. Z. Bouchal, Opt. Commun. 210, 155 (2002).

6. S. Vyas, Y. Kozawa, and S. Sata, J. Opt. Soc. Am. A 28, 837 (2011).

7. V. Garcés-Chávez, D. McGloin, H. Melville, W. Sibbett, and K. Dholakia, Nature 419, 145 (2002).

8. J. Baumgartl, M. Mazilu, and K. Dholakia, Nat. Photonics 2, 675 (2008).

9. J. Durnin, J. Opt. Soc. Am. A 4, 651 (1987).

10. P. Zhang, Y. Hu, T. Li, D. Cannan, X. Yin, R. Morandotti, Z. Chen, and X. Zhang, Phys. Rev. Lett. 109, 193901 (2012).

11. C. Alonzo, P. Rodrigo, and J. Glückstad, Opt. Express 13, 1749 (2005).

12. V. R. Daria, D. Z. Palima, and J. Glückstad, Opt. Express 19, 476 (2011).

13. V. R. Daria, D. Z. Palima, and J. Glückstad, in The Angular Momentum of Light, D. L. Andrew and M. Babiker, eds. (Cambridge University, 2012), Chap. 14.

14. M. Padgett and R. Bowman, Nat. Photonics 5, 343 (2011).

15. S. H. Tao, W. M. Lee, and X. Yuan, Appl. Opt. 43, 122 (2004).

16. N. Hermosa and C. O. Manaois, Opt. Commun. 271, 178 (2007).

17. L. C. Thomson and J. Courtial, Opt. Commun. 281, 1217 (2008).

18. A. Vasara, J. Turunen, and A. Friberg, J. Opt. Soc. Am. A 6, 1748 (1989).

19. N. Chattrapiban, E. Rogers, D. Cofield, W. Hill III, and R. Roy, Opt. Lett. 28, 2183 (2003).

20. J. W. Goodman, Introduction to Fourier Optics, 3rd ed. (Roberts \& Company, 2005).

21. N. Matsumoto, T. Ando, T. Inoue, Y. Ohtake, N. Fukuchi, and T. Hara, J. Opt. Soc. Am. A 25, 1642 (2008).

22. This formula comes from the maximum propagation distance of a BB [18]. For HCOBs, the slope of the cone depends on $\ell$, and the smallest value of the propagation distance is at its steepest slope. The steepest slope thus defines the propagation distance of the HCOBs.

23. L. Allen, M. W. Beijersbergen, R. J. C. Spreeuw, and J. P. Woerdman, Phys. Rev. A 45, 8185 (1992).

24. A. Bekshaev and M. Soskin, Opt. Lett. 31, 2199 (2006). 